\title{
Bacteria Contamination and Distribution in Patient Care Environment at Mbagathi Hospital Surgical and New-born Units
}

\author{
Enock Ongeri Magori ${ }^{1 *}$, Stanley Kamwati Kang'ethe², Kennedy Kuria Muna ${ }^{3}$, \\ Geoff rey Omuse ${ }^{4}$ and Gunturu Revathi ${ }^{4}$ \\ ${ }^{1}$ Mount Kenya University/Aga khan University P.O BOX 342-0100, Thika, Kenya \\ ${ }^{2}$ Mount Kenya University P.O BOX 342-0100, Thika, Kenya \\ ${ }^{3}$ Muranga University P.O BOX 75-0100, Muranga, Kenya \\ ${ }^{4}$ Aga Khan University Hospital P.O BOX 30270-0100, Nairobi, Kenya
}

\begin{abstract}
Background: Hospital environment can serve as an important reservoir and thus a critical element in the transmission of bacterial infections especially in critical care settings such as Surgical and new-born units. Contact with contaminated surfaces may lead to Hospital Acquired Infections (HAIs) among healthcare workers, visitors and patients. Further, HAIs may contribute to the spread of drug-resistant bacterial infections. This study sought to determine bacteriological characteristics and distribution in patient care environmental surfaces (Surgical and new-born units) at Mbagathi hospital Nairobi Kenya.

Methods: A total of 700 samples were obtained from different surfaces: beds, bedside tables, sink taps handle, door handles, nursery incubators, paediatric weighing scale and new-born resuscitation machine by means of repeated screens over a period of three months. Microbiological isolation and identification were done by following standard laboratory methods established at Aga Khan University Hospital. Antibiotic susceptibility testing was carried out by using Vitek 2 system.

Result: Five bacterial genera were isolated, S. Aureus was 3\% (19/700), E. coli 0.9\% (6/700), Acinetobacter species was 1.4\% (10/700), Pseudomonas species $0.1 \%$ (1/700) and coagulase negative staphylococcus (CoNS) $13.0 \%$ (88/700). Trimethoprim/Sulfamethoxazole and Benzylpenicillin were the most resistant antimicrobial agents while Oxacillin, Cefoxitin, Levofloxacin, Linezolid was most sensitive.

Conclusion: All S. aureus were methicillin sensitive (MSSA). Patient beds surfaces were the most contaminated among the selected items while the nursery incubator was the least contaminated.
\end{abstract}

Keywords: Hospital Environment, Bacterial Contamination, Surfaces, Surgical, New-Born Unit

\section{Introduction}

Microorganisms are known to survive on inanimate surfaces for a long period ${ }^{[1]}$ and it is more important in healthcare setting environment where patients with immunodeficiency are at a higher risk of contracting hospital acquired infections. Fomites are items that become contaminated with pathogenic organisms and act as vectors in their transmission. Pathogens are shed from different body secretions during and after the course of infections. Fomites include patient care items and environment surfaces. Surface colonization of a variety of inanimate items in a hospital facility by microorganisms has been reported as a possible route for the transmission of many disease-causing organisms. [2] Transmission of these pathogens is by direct or indirect contact with colonized or infected patients and contaminated instruments in the hospital. ${ }^{[3]}$ Mbagathi County Hospital is the largest referral hospital among the devolved units in Nairobi City County taking care of up to 800 inpatients every month with almost 2500 workers and likewise accepting at least 2300 guests of patients every day.
This populace makes it possible for contamination of highly touched areas.

The World Health Organization in 2014 acknowledged that antimicrobial resistance is a serious universal problematic area to public health. The most common multi resistant pathogens that have been documented are; S. aureus, E. coli, P. aeruginosa, Enterococcus species, Acinetobacter species, $K$. pneumonia which are frequently associated with skin and soft tissue infections. ${ }^{[4]}$ The same organisms have been isolated from fomites from hospital environment especially patient zone environment. ${ }^{[5]}$ Multidrug resistant organisms limit the curative options thus creating social and economic burden to healthcare system. These pathogens are responsible for disease, death and antimicrobial resistance in advanced and emerging developing countries. [5] Medical microbiology laboratories in developing countries especially in Kenya are sparingly spread, poorly equipped because they are inadequately financed. These challenges the Medical laboratories encounter pose a great 
danger and it is risk at this era of emergence of multidrug resistance organisms.

There is paucity of data on the prevalence of nosocomial infections and degree of bacterial contamination of mostly touched item surfaces in patient care environment and there are no records of the situation in Nairobi County mainly Mbagathi hospital. The previous studies that have been done on patients' clinical samples in chosen hospitals in Nairobi City County, MRSA was regularly isolated from public healthcare amenities attending economically underprivileged Nairobi populace such as those residing in metropolitan informal settlements. ${ }^{[6]}$ Other reports indicate there is transmission of MRSA and MSSA in public healthcare facilities in Kenya. ${ }^{[7]}$

\section{Materials and Methods}

This study was conducted at Mbagathi County Hospital located in Dagoretti sub-county of Nairobi City County in Kenya between May and July 2020. The hospital has a 200 bed-capacity with approximately 800 admissions monthly and 1000 outpatient's visits per day where around 300 healthcare practitioners attend them. In general ward at Mbagathi Hospital, floors are mopped twice in a day by using detergent solutions and in corridors floors they are scrubbed by using mopping machines weekly. Working staff tables and benches are wiped once daily by using sodium hypochlorite solutions. There is no protocol of cleaning/ disinfecting surfaces of bed rails, nursery incubator, bed side table, water sink handle, ward door handle, paediatric weighing scale, ward drug trolley, new-born and resuscitation machine. The study was approved by Mount Kenya University, National Commission for Science, Technology and Innovation ethical committees. Authorization was also granted by the Mbagathi Hospital and Aga Khan University Hospital ethical committee.

\section{Specimen Collection}

A total of 700 samples were collected from different item surfaces within Surgical and New-born units. Samples were collected from bed rails, nursery incubator, bed side table, water sink handle, ward door handle, paediatric weighing scale, ward drug trolley, new-born and resuscitation machine. Samples from chosen surfaces were collected by rubbing and rotating sterile cotton swabs moistened with $0.9 \%$ normal saline firmly over the entire target surface which were conveniently selected from surgical and newborn units. These sites were more accessible and mostly used by patients, visitors and healthcare workers. These units were selected because of patient load and likelihood of long stay in the hospital.
Isolation and identification of bacterial isolates, The swabs upon collection and well labelled, they were transported to Aga Khan University Hospital Laboratory in amies transport media. The specimens were inoculated within two hours after collection onto 5\% sheep blood agar by streaking the agar surfaces with wire loop then incubated aerobically at $37^{\circ} \mathrm{C}$ for up to 48 hours. After the end of incubation period, the culture plates were examined for bacterial growth and their morphological features of the organisms on the agar used. The identification of culture isolates was done according to standard operating procedures adapted at Aga Khan University Hospital. Conventional identification criteria included Gram staining, catalase, coagulase and oxidase tests. Further identification was performed by using the Vitek 2 system.

\section{Antibiotic susceptibility test}

An automated Antibiotic susceptibility to a standard panel of antibiotic was performed by Vitek 2 machine, version 4.01 (Biomerieux, Mercy-I' Etoile France) an automated system for bacterial identification which performs antibiotic susceptibility by using broth dilution. Also, Kirby disc diffusion method was used. Bacterial isolates that was resistant to three or more drug classes were categorized as multidrug resistant organisms (MDR)

\section{Quality control}

Culture media were incubated without inoculum to check the sterility of the culture media. Standard organisms were inoculated on 5\%sheep blood agar and MacConkey to check their growth support. Standard organism used as control for identification and susceptibility are; Staphylococcus aureus (ATCC ID: 25923 and ATCC AST: 29213) Escherichia coli (ATCC 25922), Pseudomonas aeruginosa (ATCC 27853).

\section{Result}

Out of 700 collected samples from various item surfaces, growth was obtained in 63\% (442/700) samples of which $18 \%(124 / 700)$ were identified as clinical pathogens. $37 \%$ $(258 / 700)$ of the samples, there were no growth obtained. Various bacterial flora was isolated from different surfaces. The bacterial contamination in the beds, incubators, bedside tables and ward drug trolleys was 30.7\%, 2.4\%, $29.5 \%$ and $32 \%$ respectively.

Based on sample item sites, for bed, prevalence was highest in the right-hand side (48\%) and lowest in the head and bottom sides $(28 \%)$. Prevalence in incubators was highest in the top outside (12\%). Prevalence was highest in the top $(20 \%)$ in bedside tables, handle $(20 \%)$ in ward doors and handle $(10 \%)$ in water sinks. 
The study found that five bacterial genera were isolated (S. aureus, E. coli, Acinetobacter spp, Pseudomonas spp, CoNS). It was determined that the prevalence of $S$. aureus bacterial contamination in patient care environment at Mbagathi hospital surgical and new-born unit was 3\%,E. coli $(0.9 \%)$, Acinetobacter spp (1.4\%), Pseudomonas spp $(0.1 \%)$, while the most dominant contaminant was CoNS (13\%). Most $S$. aureus isolates were obtained from bed rails $(12 / 19)$, ward door handles (3/19), drug trolley (1/19) and bedside table (3/19). All the $S$. aureus were MSSA as shown on table 4 . Out of $19 \mathrm{~S}$. aureus isolated, 11 were multidrug resistant (table 5). For Gram negative antimicrobial resistance pattern, it is demonstrated in table 4-7.

\section{Discussion}

The study found that item surfaces in surgical and Newborn units that are commonly used by patients, visitors and Healthcare workers were contaminated with potential pathogens. The role of the items on how they are involved in infection transmission was not determined in this study. However, the isolation of $S$. aureus, Coagulase negative Staphylococci, E. coli, Pseudomonas species, Acinetobacter species makes it possible for the transmission of Hospital Acquired Infection. Nosocomial pathogens if there is no continuous disinfection of surfaces would be the constant source of transmission as these organisms can survive on item surfaces for months.

Table 1: Bacterial distribution pattern on ward items surfaces.

\begin{tabular}{|c|c|c|c|c|c|c|c|}
\hline & S. aureus & E. coli & $\begin{array}{c}\text { Acinetobacter } \\
\text { spp. }\end{array}$ & $\begin{array}{c}\text { Pseudomonas } \\
\text { spp. }\end{array}$ & CoNS & Total & $P$ value \\
\hline Surgical bed & $12(14.0)$ & $5(8.1)$ & $7(5.5)$ & $1(1.2)$ & $61(71.0)$ & $86(100.0)$ & \\
\hline Incubator NBU & $0(0.0)$ & $0(0.0)$ & $0(0.0)$ & $0(0.0)$ & $2(100.0)$ & $2(100.0)$ & \\
\hline Bedside table & $3(33.3)$ & 1(11.1) & $3(33.3)$ & $0(0.0)$ & $2(22.3)$ & $9(100.0)$ & .845 \\
\hline Water sink & $0(0.0)$ & $0(0.0)$ & $0(0.0)$ & $0(0.0)$ & $1(100.0)$ & $1(100.0)$ & \\
\hline Ward door & $3(75.0)$ & $0(0.0)$ & $0(0.0)$ & $0(0.0)$ & $1(25.0)$ & $4(100.0)$ & \\
\hline Ward trolley & $1(33.3)$ & $0(0.0)$ & $0(0.0)$ & $0(0.0)$ & $2(66.7)$ & $3(100.0)$ & \\
\hline
\end{tabular}

Table 2: Bacterial distribution pattern in ward item sites.

\begin{tabular}{|c|c|c|c|c|c|c|c|c|}
\hline & & $\begin{array}{c}\text { S. } \\
\text { aureus }\end{array}$ & E. coli & $\begin{array}{c}\text { Acinetobacter } \\
\text { spp. }\end{array}$ & $\begin{array}{c}\text { Pseudomonas } \\
\text { spp. }\end{array}$ & Cons & Total & $\begin{array}{c}P \\
\text { value }\end{array}$ \\
\hline \multirow{4}{*}{ Bed sites } & Head & $2(12.0)$ & $2(12.0)$ & $0(0.0)$ & $0(0.0)$ & $13(76.0)$ & $17(100.0)$ & \multirow{4}{*}{.345} \\
\hline & Bottom & $4(20.0)$ & $1(5.0)$ & $2(10.0)$ & $0(0.0)$ & $13(65.0)$ & $19(100.0$ & \\
\hline & Left hand side & $3(12.0)$ & $2(8.0)$ & $2(8.0)$ & $0(0.0)$ & $18(72.0)$ & $25(100.0)$ & \\
\hline & Right hand side & $4(14.0)$ & $0(0.0)$ & $3(10.0)$ & $1(3.0)$ & $21(73.0)$ & $29(100.0)$ & \\
\hline Incubator sites & Top outside & $0(0.0)$ & $0(0.0)$ & $1(20.0)$ & $0(0.0)$ & $4(80.0)$ & $5(100.0)$ & - \\
\hline \multirow{2}{*}{ Bedside table } & Top & $3(18.0)$ & $1(6.0)$ & $2(13.0)$ & $0(0.0)$ & $10(63.0)$ & $16(100.0)$ & \multirow[b]{2}{*}{-} \\
\hline & Handle & $0(0.0)$ & $0(0.0)$ & $0(0.0)$ & $0(0.0)$ & $5(100.0)$ & $5(100.0)$ & \\
\hline Water sink & Handle & $0(0.0)$ & $0(0.0)$ & $0(0.0)$ & $0(0.0)$ & $2(100.0)$ & $2(100.0)$ & - \\
\hline Ward door & Handle & $4(80.0)$ & $0(0.0)$ & $0(0.0)$ & $0(0.0)$ & $2(20.0)$ & $6(100.0)$ & - \\
\hline
\end{tabular}

Table 3: Antimicrobial sensitivity patterns of isolated $S$. aureus species.

\begin{tabular}{|l|c|c|c|}
\hline Antimicrobial agent & Resistant (N) & Sensitive (N) & Total (N) \\
\hline Linezolid & 0 & 19 & 19 \\
\hline Trimethoprim/Sulfamethoxazole & 14 & 5 & 19 \\
\hline Benzylpenicillin & 14 & 5 & 19 \\
\hline Erythromycin & 7 & 12 & 19 \\
\hline Clindamycin & 4 & 14 & 19 \\
\hline Gentamicin & 0 & 19 & 19 \\
\hline
\end{tabular}




\begin{tabular}{|l|c|c|c|}
\hline Antimicrobial agent & Resistant (N) & Sensitive (N) & Total (N) \\
\hline Oxacillin & 0 & 19 & 19 \\
\hline Cefoxitin & 0 & 19 & 19 \\
\hline Levofloxacin & 0 & 19 & 19 \\
\hline Tetracycline & 6 & 13 & 19 \\
\hline Rifampicin & 6 & 13 & 19 \\
\hline
\end{tabular}

Table 4: Multidrug resistance nature of $S$. aureus isolates in patient care environment.

\begin{tabular}{|l|c|}
\hline Antimicrobial & No. Resistant \\
\hline $\begin{array}{l}\text { Trimethoprim/Sulfamethoxazole + Benzylpenicillin + } \\
\text { Erythromycin + Clindamycin }\end{array}$ & 4 \\
\hline $\begin{array}{l}\text { Trimethoprim/Sulfamethoxazole + Benzylpenicillin + } \\
\text { Rifampicin }\end{array}$ & 4 \\
\hline $\begin{array}{l}\text { Trimethoprim/Sulfamethoxazole + Benzylpenicillin + } \\
\text { Tetracycline }\end{array}$ & 3 \\
\hline Total & $\mathbf{1 1}$ \\
\hline
\end{tabular}

Table 5: Antimicrobial sensitivity patterns of isolated $E$. coli.

\begin{tabular}{|l|c|c|c|}
\hline Antimicrobial agent & Resistant (N) & Sensitive (N) & Total (N) \\
\hline Ciprofloxacin & 2 & 4 & 6 \\
\hline Trimethoprim/Sulfamethoxazole & 3 & 3 & 6 \\
\hline Ceftazidime & 2 & 4 & 6 \\
\hline Gentamicin & 2 & 4 & 6 \\
\hline Meropenem & 1 & 5 & 6 \\
\hline Ceftriaxone & 3 & 3 & 6 \\
\hline
\end{tabular}

Table 6: Antimicrobial sensitivity patterns of isolated Acinetobacter species.

\begin{tabular}{|l|c|c|c|}
\hline Antimicrobial agent & Resistant (N) & Sensitive (N) & Total (N) \\
\hline Imipenem & 1 & 9 & 10 \\
\hline Cefotaxime & 6 & 4 & 10 \\
\hline Clotrimoxazole & 5 & 5 & 10 \\
\hline Ciprofloxacin & 4 & 6 & 10 \\
\hline Ceftazidine & 6 & 4 & 10 \\
Gentamicin & 4 & 6 & 10 \\
\hline
\end{tabular}

Table 7: Antimicrobial sensitivity patterns of isolated Pseudomonas species.

\begin{tabular}{|l|l|l|l|}
\hline Antimicrobial agent & Resistant (N) & Sensitive (N) & Total (N) \\
\hline Ceftriaxone & 0 & 1 & 1 \\
\hline Trimethoprim/Sulfamethoxazole & 1 & 0 & 1 \\
\hline Gentamicin & 0 & 1 & 1 \\
\hline Ciprofloxacin & 0 & 1 & 1 \\
\hline Meropenem & 0 & 1 & 1 \\
\hline Ceftazidine & 0 & 1 & 1 \\
\hline
\end{tabular}


The findings when compared to other studies elsewhere, the prevalence rate in this study was $18 \%$ slightly lower than that reported by Nwankwo ${ }^{[8]}$ at Murtala Mohammed Specialist Hospital in Nigeria. The prevalence rate by Nankwo was found to be $23.33 \%(95 \% \mathrm{CI}=20.28$ $-26.71 \%$ ). However, Ensayef ${ }^{[9]}$ found the prevalence to be even lower at Al Imam Ali Hospital in Bagdad 4\%. However, this prevalence was also lower than that reported byAl-Zoubi, ${ }^{[10]}$ whose prevalence was $98.7 \%$ at San Vicente Foundation, a teaching hospital in Columbia and Gondar University Hospital in which 83.1\% (95\% CI = $68.78-99.52 \%$ ) of inanimate objects were contaminated.

The study determined that the prevalence of $S$. aureus bacterial contamination in patient care environment at Mbagathi hospital surgical and new-born unit was 3\%. This prevalence was lower than that recorded by Segujja ${ }^{[11]}$ who found the $167(57.59 \%, \mathrm{CI}=49.18-67.01)$ harboured bacterial pathogens, where $S$. aureus prevalence was $25.75 \%$. The prevalence of E. coli was $18.55 \%$, a higher prevalence than that of Mbagathi hospital surgical and new-born unit, which was found to be $0.9 \%$. However, the current study did not find existence of some bacteria such E. faecalis, S. epidermidis, P. mirabilis, Bacillus species and $S$. saprophyticus which were prevalent in Segujja ${ }^{[11]}$ study.

The study found that CoNS (13.0\%) and Staphylococcus aureus (3\%) were the most frequently isolated pathogen across the hospital surfaces, a consistent result with other findings from different studies and authors across the world. ${ }^{[12,13]}$ In this study, CoNS were the most prevalent in all item sites. S. Aureus were present in surgical beds, ward doors, bedside table and drug trolleys. The other type of bacteria did not cut across all surfaces, and only existed in one or two surfaces. In this regard, E. coli were only present in surgical beds. Acinetobacter species were present in surgical beds bedside table, nursery incubator. Pseudomonas species were only present in surgical beds.

The prevalence of Acinetobacter species at Mbagathi hospital surgical and new-born unit was $1.4 \%$. In contrast to these findings, Carvalheira ${ }^{[14]}$ found that the most common species were Acinetobacter guillouiae with a prevalence of $34.9 \%$. In addition, Banerjee et al. (2018) found a high prevalence of Acinetobacter species associated with HCAI in the adult ICU of a tertiary care hospital in Varanasi, north India. Moreover, ${ }^{[15]}$ also revealed that Acinetobacter species. were the significant major pathogen against other Gram-negative organisms, which is the same finding to the present study. The prevalence of Pseudomonas species at Mbagathi hospital surgical and new-born unit was found to be $0.1 \%$. These findings are different from those of
Banerjee ${ }^{[15]}$ who found the prevalence of Pseudomonas spp to be $10.17 \%$ in Intensive Care Unit of a Tertiary Care Hospital, Varanasi, India.

The prevalence of contamination in the beds, incubators, bedside tables and ward drug trolleys was 30.7\%, 2.4\%, $29.5 \%$ and $7 \%$ respectively. The prevalence of bacterial contamination was therefore highest in the beds. The findings when compared to other studies agree with those of Zazouli ${ }^{[16]}$ who found a significant prevalence of contamination in hospital beds. The overall contamination was $40 \%$, which is slightly higher than that found in the beds in this study $(36.5 \%)$. Hassan ${ }^{[17]}$ also found that the most regularly contaminated surfaces were the bed tops, and bed sides. These findings are also true as postulated in a study by Saka ${ }^{[18]}$.

\section{Conclusion}

From the findings obtained in this study, the study concludes that bacterial contamination at Mbagathi hospital surgical and new-born unit on item surfaces that are frequently used by healthcare works, visitors and patients is $18 \%$. Specifically, the prevalence of $S$. aureus was $3 \%, E$. coli $(0.9 \%)$, Acinetobacter spp $(1.4 \%)$, Pseudomonas spp $(0.1 \%)$, while the most dominant contamination was CoNS $(13.0 \%)$. The isolation of pathogens from fomites demonstrates poor hand hygiene among visitors, healthcare workers and patients. These potential pathogens that were isolated from mostly touched surfaces signify the importance of establishing the cleaning protocol and disinfection of these sites. There is need to emphasize the importance of hand hygiene and decontamination of these sites regularly.

\section{Acknowledgements}

We thank Germain Research Foundation for funding this study.We also thank microbiology staff of Aga Khan University Hospital for their technical support. We thank Mount Kenya University, Mbagathi Hospital and Aga Khan University Hospital for giving us a chance to study and giving us a chance to collect samples and carry out our experiments.

\section{Funding}

This project was funded by -DFG grant

ZI-665/3-0(20028-2022). Principal Investigator-Gunturu Revathi of Aga Khan University, Nairobi.

\section{Competing Interests}

None declared. 


\section{Reference}

1. Russotto, V., Cortegiani, A., Raineri, S. M., Iozzo, P., Gregoretti, C., \& Giarratano, A. (2017). What is the risk of acquiring bacteria from prior intensive care unit bed occupants?. Critical Care, 21(1), 55-62.

2. Olise, C. C., Simon-Oke, I. A., \& Akumeh, D. D. (2018). Clinical isolates resistance to commonly used antibiotics: A concern in healthcare setting. International Journey Public Health Safe, 3(150), 2-4.

3. Facciola, A., Pellicano, G. F., Visalli, G., Paolucci, I. A., Rullo, E. V., Ceccarelli, M., ..., \& La Fauci, V. (2019). The role of the hospital environment in the healthcare-associated infections: a general review of the literature. European review for medical and pharmacological sciences, 23(1), 1266-1278.

4. Pendleton, J. N., Gorman, S. P., \& Gilmore, B. F. (2013). Clinical relevance of the ESKAPE pathogens. Expert review of anti-infective therapy, 11(3), 297-308.

5. Bhatta, D. R., Hamal, D., Shrestha, R., Subramanya, S. H., Baral, N., Singh, R. K., ... \& Gokhale, S. (2018). Bacterial contamination of frequently touched objects in a tertiary care hospital of Pokhara, Nepal: how safe are our hands? Antimicrobial Resistance \& Infection Control, 7(1), 97.

6. Maina, E. K., Kiiyukia, C., Wamae, C. N., Waiyaki, P. G., \& Kariuki, S. (2013). Characterization of methicillin-resistant Staphylococcus aureus from skin and soft tissue infections in patients in Nairobi, Kenya. International journal of infectious diseases, 17(2), e115-e119.

7. Oosterik, L. H., Peeters, L., Mutuku, I., Goddeeris, B. M., \& Butaye, P. (2014). Susceptibility of avian pathogenic Escherichia coli from laying hens in Belgium to antibiotics and disinfectants and integron prevalence. Avian diseases, 58(2), 271-278

8. Nwankwo, E. (2012). Isolation of pathogenic bacteria from fomites in the operating rooms of a specialist hospital in Kano, North-western Nigeria. Pan African Medical Journal, 12(1), 12-19.

9. Ensayef, S., Al Shalchi, S., \& Sabbar, M. (2009). Microbial contamination in the operating theatre: a study in a hospital in Baghdad. EMHJ-Eastern Mediterranean Health Journal, 15(1), 219-223.
10. Al-Zoubi, M. S., Al-Tayyar, I. A., Hussein, E., Al Jabali, A., \& Khudairat, S. (2015). Antimicrobial susceptibility pattern

of Staphylococcus aureus isolated from clinical specimens in Northern area of Jordan. Iranian journal of microbiology, 7(5), 265-281.

11. Segujja, F., Mwambi, B., Drago, C. K., Lubowa, N. M., Mugambwa, J., \&Wabuyi, P. (2016). Characterization and antimicrobial susceptibility patterns of isolates from ward fomites. Kampala, Uganda: Makerere University.

12. Feglo, P., \&Afriyie-Asante, A. (2014). Environmental impact on postoperative wound infections in a privately owned hospital in Ghana. African Journal of Microbiology Research, 8(15), 1620-1626.

13. Yallew, W. W., Kumie, A., \&Yehuala, F. M. (2016). Point prevalence of hospital-acquired infections in two teaching hospitals of Amhara region in Ethiopia. Drug, healthcare and patient safety, 8(1), 71-79.

14. Carvalheira, A., Casquete, R., Silva, J., \& Teixeira, P. (2017). Prevalence and antimicrobial susceptibility of Acinetobacter spp. isolated from meat. International journal of food microbiology, 243(1), 58-63.

15. Banerjee, T., Mishra, A., Das, A., Sharma, S., Barman, H., \& Yadav, G. (2018). High prevalence and endemicity of multidrug resistant Acinetobacter spp. in intensive care unit of a tertiary care Hospital, Varanasi, India. Journal of pathogens, 2018(1), 1-9.

16. Zazouli, M. A., Yazdani-charati, J., Ahanjan, M., \&Eslamifar, M. (2017). Bacterial contamination of environmental surfaces in two educational hospitals under the auspices of Mazandaran University of Medical Sciences. Journal of Health in the Field, 3(1), 22-29.

17. Hassan, M. Z., Sturm-Ramirez, K., Rahman, M. Z., Hossain, K., Aleem, M. A., Bhuiyan, M. U., ... \& Gurley, E. S. (2019). Contamination of hospital surfaces with respiratory pathogens in Bangladesh. PloS one, 14(10), e0224065.

18. Saka, K. H., Akanbi, A. A., Obasa, T. O., Raheem, R. A., \&Oshodi, A. J. (2017). Bacterial Contamination of Hospital Surfaces According to Material Make, Last Time of Contact and Last Time of Cleaning/Disinfection. Journal ofBacterialParasitology, 8(03), 8-11.

*Corresponding author:

Enock Magori, Mount Kenya University/Aga khan University P.O BOX 342-0100, Thika, Kenya

Phone: +254713489101

Email: Enockongeri2010@gmail.com

Date of Submission : 04/12/2020

Date of Acceptance : 23/02/2021

Financial or other Competing Interests: None. 